INTERNATIONAL JOURNAL OF SCIENTIFIC RESEARCH

ORAL CANDIDA CARRIAGE INCLUDING SPECIATION, BIOFILM PRODUCTION AND CORRELATION WITH OCCURRENCE OF DENTAL CARIES IN STUDENT POPULATION

\title{
Microbiology
}

\section{Sumi Nandwani}

Professor and Chief Consultant, Microbiology, Super Speciality Paediatric Hospital and Post Graduate Teaching Institute, Noida, Uttar Pradesh -201303

\section{ABSTRACT}

The study was done to establish oral carriage of Candida, speciation, assay biofilm production and correlate the oral Candida carriage with occurrence of dental caries in students. Swab samples from oral cavity were collected from 206 students and were cultured for yeasts. Isolated yeasts were identified and detected for Biofilm production.

Results: Overall Candida carriage was found in 90 of the 206 students ( 75 with caries). The rate of Candida carriage in students with caries (64\%) was significantly higher as compared to overall carriage in students (43.7\%). A total of 123 Candida strains were isolated including C albicans $(63 \%)$, C tropicalis (23\%), C. parapsilosis (6\%), C. glabrata (4\%) and C. krusei (4\%). Biofilm production by C albicans was less frequent (42. $85 \%)$ than that by non-C albicans $(63.33 \%)$. and was significantly higher in students with caries than from without caries.

Conclusion: We found a significant risk of dental caries with oral Candida carriage rates in student population. Candida albicans was the most common species isolated. Biofilm production was implicated in Candida virulence and was more important for non- C.albicans than C.albicans strains to establish infections.

\section{KEYWORDS}

Biofilm production; Candida; Caries; Oral Carriage; Students.

\section{INTRODUCTION}

Apart from bacteria, the importance of the presence of yeasts in the oral cavity and the incidence of dental caries have been demonstrated for adults and for children in earlier studies. ${ }^{1}$ Although mutans streptococci and lactobacilli are considered to be the main aetiological agents of human dental caries, fungal microflora has been found to be involved by numerous studies suggesting a correlation between high prevalence of Candida spp. and the development of active carious lesions, however it's actual role as a risk factor has yet to be completely clarified.

Candida albicans is frequently carried in the oral cavity without causing disease ( $20 \%$ to $40 \%$ but asymptomatic carriage may place some individuals at higher risk of complications if they become immunosuppressed. ${ }^{3}$ The genus Candida includes several species of which Candida albicans is by far the most common species causing infections in humans. The emergence of non- albicans Candida spp. as significant pathogens has however been well recognized during the past decade. ${ }^{4}$ All Candida spp. have been shown to cause a similar spectrum of disease ranging from oral thrush to invasive disease, yet differences in disease severity and susceptibility to different antifungal agents have been reported. ${ }^{4}$ Candida spp. identification is therefore important for successful management.

Despite the potential relevance of C. albicans carriage, little is known about carriage patterns in student population. Hence, the purpose of the present study was to analyse oral carriage of different Candida species in students and to correlate their carriage with dental caries.

In the present study we have also aimed at demonstrating biofilm formation by candida spp isolated which may be a reflection of their pathogenic potential. Candida strains possess a number of virulence factors; one amongst them is biofilm (slime) production. Biofilms are the structured microbial communities that are attached and encased in a matrix of exopolymeric material increasing candida adherence ability and colonization and infection of host tissues leading to development of clinical infection particularly in the oral cavity. .

\section{AIMS \& OBJECTIVES:}

1. To analyse oral carriage of different Candida species in students.

2. To correlate their carriage with dental caries in them.

3. Demonstrating biofilm formation by Candida spp. isolated

\section{METHODS:}

The samples for the study were collected from the pharynx, supragingival plaques and carious lesions using sterile cotton swabs in 206 dental students with an average age of 28 years persuing B.D.S. course $\left(2^{\text {nd }}\right.$ year)in a private dental and medical college in Delhi, NCR Region over a period of 06 months with prior informed consent. All were clinically healthy with no signs of systemic or any other illness.

All samples were cultured directly on Sabourauds Dextrose Agar slopes with chloramphenicol and incubated at $37{ }^{\circ} \mathrm{C}$ for 3 days. Macroscopic (creamy moist colonies) and microscopic (yeast cells, pseudohyphae and blastospores) examination of the growth verified the diagnosis of candidiasis ${ }^{4}$ Species identification of Candida isolated was done by performing germ tube, sugar fermentation and assimilation tests.

Further subculture of the obtained colonies was done on Cornmeal agar plates and Hi-Crome Candida Differential Agar M1297A on which different Candida species produce different colour after incubation at $30^{\circ} \mathrm{C}$ for $24-48$ hours.

Virulence was determined as adherence capacity by biofilm production by the species. For measuring the Biofilm (slime) production, a saline suspension of 24-hour growth of organisms from the SDA plate was made and turbidity adjusted to 0.5 MacFarland standard to contain $3 \times 10^{7} \mathrm{cfu} / \mathrm{ml} .20 \mu \mathrm{l}$ of this suspension was inoculated into wells of microtiter plate to which $180 \mu \mathrm{l}$ Sabouraud's Dextrose broth was added. The plates were incubated at $37^{\circ} \mathrm{C}$ for $24 \mathrm{~h}$ after which the wells were washed twice with phosphate buffered saline and finally $200 \mu \mathrm{l}$ of PBS was added to each well. Biofilm production was measured by spectrophotometric reading at $405 \mathrm{~nm}$ and quantified by measuring the percent transmittance with microtitre plate reader. Biofilm production was scored as negative, weak positive $(1+)$, moderate positive $(2+)$ or strong positive $(3+) .{ }^{5,10}$ The prevalence of Candida carriage was calculated using the chi- square test. The levels of significance were fixed at $\mathrm{p}<0.05$.

\section{RESULTS:}

The overall Candida carriage was found in 90 out of 206 students with 75 students having caries. Out of 90 students, Candida was isolated from single material sample in 61 students and in the rest, 29 Candida was isolated from 2 ( 25 students) or 3 materials ( 4 students).

A total of 123 Candida strains were isolated of which 61(49.6\%) were from supragingival plaques, $48(39 \%)$ were from carious lesions (out of 75$)$ and $14(11.4 \%)$ were from pharyngeal swabs.

Candida was isolated in 48 out of 75 (64\%) students with caries, the rate being significantly higher as compared to overall number of students with candidial carriage being 90 out of 206 (43.7\%) (p = $0.0026)$.

Out of 123 candida strains isolated, the species identified were $\mathrm{C}$ albicans $(63 \%), \mathrm{C}$ tropicalis $(23 \%), \mathrm{C}$. parapsilosis $(6 \%)$, C. glabrata $(4 \%)$ and C. krusei $(4 \%)$ (Table 1$)$.

Amongst the 123 Candida strains, biofilm production was found in 61 strains $(49.6 \%)$. Biofilm production by $\mathrm{C}$ albicans was less frequent (33 out of 78 strains, $42.3 \%$ ) than that by non-C albicans ( 28 out 45 strains, $63.22 \%$ ). Strong biofilm production was found in C. krusei (4 out of 5 strains showed $4+$ biofilm production), weak in C. parapsilosis 
(5out of 7 showed no biofilm production and 1 each showed $2+$ and $1+$ biofilm production).

Biofilm production among candida strains isolated from student with caries (mean $0.293 \pm 0.046$ ) was statistically significantly higher $(\mathrm{p}=$ 0.05 ) than in those obtained from students without caries (mean 0.328 $\pm 0.067)$. (Table 2$)$

\section{DISCUSSION:}

Our own observations showed oral Candida isolation rate in students with caries as significantly higher as compared to overall number of students with candidial carriage in oral cavity indicating that there is an increased risk of dental caries with oral Candida carriage rates in student population. This is in compliance with other studies implicating oral Candida carriage in pathogenesis of plaque and caries and especially in the progression of caries lesions in children. ${ }^{2,11,12}$ The Candida spp. are capable of colonizing the hard surface of the teeth, invading the dentinal tubules, participating in formation of microbial biofilm and producing large amount of acids responsible for demineralization of tooth enamel and dissolution of hydroxyapatite. ${ }^{2,13}$ The results obtained suggest that anti-caries treatment alone is not sufficient for resolving dental decay, and should be supplemented with additional therapy with antifungal mouthwashes in particular when yeasts are present. A similar inference has been made by a study where oral Candida carriage was found to be related to caries in children and carriage rates were reduced with use of antifungal oral rinses to eliminate any Candida spp. reservoir to prevent reinfection.

According to most publications, Candida albicans is the most frequently encountered species in both oral carriage and oral candidiasis as was found in our study.$^{1,4,12} \mathrm{~A}$ study done earlier in China however found that frequency of Candida species varied with age group. It stated that with increasing age, the frequency of C. albicans decreases and non-C. albicans yeasts increases.$^{14}$ Emergence of non-C. albicans strains as significant pathogens especially in immuno compromised patients with varied clinical manifestations has also been reported in other studies."

The ability of fungi to form biofilms has been associated with the ability to cause infections like oral candidiasis and bloodstream infections

In the present study, biofilm production among Candida strains isolated from students with caries was statistically significantly higher than in those obtained from students without caries implicating its production in enhancing Candida virulence which is similar to that reported in earlier studies. ${ }^{5,6,16}$

Biofilm production by $\mathrm{C}$. albicans was found to be less frequent than that by non-C. albicans species suggesting that biofilm production is more important for non- C. albicans strains and C. albicans possess mechanisms other than biofilm production, as reported in earlier studies. ${ }^{5}$

\section{CONCLUSIONS:}

The study suggests oral Candida carriage may have a role in pathogenesis of dental caries possibly by biofilm production in student population. Candida albicans was the commonest species isolated in oral cavity, however non- $\mathrm{C}$ albicans species have a higher potential for bio film production and virulence.

Table 1. Proportion Of Students With And Without Caries With Candida Carriage

\begin{tabular}{|l|l|l|l|}
\hline No. of students & Examined & With Caries & Without Caries \\
\hline Total & 206 & 75 & 131 \\
\hline Candida isolated & 90 & 48 & 42 \\
\hline
\end{tabular}

TABLE 2. Candida species isolated and biofilm production by them

\begin{tabular}{|c|c|c|c|c|c|c|c|}
\hline \multirow[t]{2}{*}{ Species } & \multirow{2}{*}{\begin{tabular}{|l|} 
No. \\
isolated
\end{tabular}} & \multicolumn{4}{|c|}{ Biofilm production } & \multirow{2}{*}{\begin{tabular}{|l} 
Biofilm \\
producers
\end{tabular}} & \multirow{2}{*}{\begin{tabular}{|l} 
Biofilm \\
Non \\
producers
\end{tabular}} \\
\hline & & $3+$ & $2+$ & $1+$ & 0 & & \\
\hline C. albicans & 78 & 2 & 11 & 20 & 45 & 33 & 45 \\
\hline C. tropicalis & 28 & 6 & 6 & 7 & 9 & 19 & 9 \\
\hline C. parapsilosis & 07 & 0 & 1 & 1 & 5 & 2 & 5 \\
\hline C. glabrata & 05 & 0 & 2 & 0 & 3 & 2 & 3 \\
\hline C. krusei & 05 & 4 & 1 & 0 & 0 & 5 & 0 \\
\hline \begin{tabular}{|l} 
Total \\
\end{tabular} & 123 & & & & & 61 & 62 \\
\hline
\end{tabular}

\section{REFERENCES:}

1. Ugun-Can B, Kadir T, Akyüz S. Oral Candidal carriage in children with and withou dental caries. Quintessence Int.2006: Dec; 41-45

2. Caterina S, Burlacchini G, Faccioni F, Zanderigo M, Bozzola N, Canepari P. Support for the role of Candida spp. in extensive caries lesions of children. New Microbiologica. 2009:32; 101-7

3. Hibino K, Wong RWK, Hag U, Samaranayake LP. The effects of orthodontic appliances on Candida in the human mouth. International Journal of Paediatric Dentistry. 2009: 19; 301-8

4. Shaheen MA and Taha M. Species Identification of Candida Isolates Obtained from Ora lesions of Hospitalized and Non Hospitalized Patients with Oral Candidiasis. Egyptian Derms or Hospitaze

5. Vinitha M Mamata B. Biofilm as virulence marker in Candida isolated from blood. World J of Medical Sceinces. 2007: 2(1); 46-8

6. Cristaine YK, Juliana PL, Valerio V \& Maria AR, Virulence factors and antifungal susceptibility of Candida albicans isolates from oral candidosis patients and contro individuals. Mycopathologia. 2006:161;219-23

7. Hazen KC, Howell SA. Fungi. Candida, Cryptococcus and other yeasts of medical importance. 114. In: Manual of clinical microbiology. Murray PR, Baron EJ, Jorgensen JH, Pfaller MA, Yolken RH, editors.8th ed., Washington, D.C., American Society fo Microbiology Press. 2003: 2; 1693-711.

8. Rippon J.W. (1988): Medical Mycology; The Pathogenic fungi and Pathogenic Actinomycetes.3rd ed.WB. Saunders Co., Philadelphia.

9. Murray MP, Zinchuk R, Larone DH. Chrom agar candida as the sole primary medium for isolation of yeasts and as a source medium for the rapid assimilation of trehalose test. J. isolation of yeasts and as a source

10. Chandra J, Kuhn DM, Mukherjee PK, Hoyer LL, McCormick T, Ghannoum MA Biofilm formation by the fungal pathogen Candida albicans: development, architecture and drug resistance. J Bacteriol 2001:183; 5385-94.

11. Hodson JJ, Craig G.T. The incidence of Candida albicans in plaques of teeth of children. The Dental Practitioner. 1972: 22(8); 296-301

12. Rozkiewicz D et al. Oral Candida albicans carriage in healthy preschool and schoo children. Advances in Medical Sciences. 2006: 51 (1); 187-90

13. Akdeniz.BG, Koparal E, Sen BH, Ates M, Denizci AA. Prevalence of candida albicans in oral cavities and root canals of children. Journal of Dentistry for children. 2002: SeptDec; $289-292$

14. Qi. G, Hu. T, Zhou.X.D. Frequency, species and molecular characterization of oral Candida in hosts of different age in China. J Oral Pathol Med. 2005: 34; 352-6

15. Malini RC, Deepthi N, Manorama D, Pradeep KV, Lakshmi S, Pushpa A. Emergence of Non- albicans Candida species and antifungal resistance in a tertiary care hospital. Jpn.J.Infect. Dis 2005:58:344-348

16. Vinitha M. D, Mamatha B. Proteinase and phospholipase activity as virulence factors in Candida species isolated from blood. Rev Iberoam Micol.2008: 25; 208-210 